\title{
INHALTSVERZEICHNIS
}

\section{BORCHERT, GÜNTER}

(Universität Hamburg, Institut für Geographie und Wirtschaftsgeographie)

Beiträge von Hamburger Geographen zur Afrikaforschung .... 1

\section{JANTZEN, GÜNTHER}

(Handelskammer Hamburg)

Zu Hamburgs West- und Ost-Afrika-Beziehungen.

Wechselwirkungen von Afrika-Wirtschaft und -Wissenschaft .. 21

WEBER, HANS

(Deutsche Erdöl-Aktiengesellschaft, Hamburg)

Hamburgs Anteil an der geologischen Erforschung Afrikas . .. 35

HölLER, ERICH

(Deutscher Wetterdienst, Seewetteramt Hamburg)

Hamburgs Beitrag zur Erforschung der klimatischen Verhält-

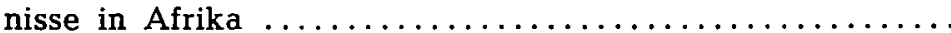

Kubiena, Walter L.

(Bundesforschungsanstalt für Forst- und Holzwirtschaft, Reinbek)

Hamburgs Beitrag zur bodenkundlichen Forschung in Afrika . .

Maydell, Hans Jürgen von

(Bundesforschungsanstalt für Forst- und Holzwirtschaft, Reinbek)

Hamburger forst- und holzwirtschaftliche Forschungen in Afrika

WALTHER, KURT

(Universität Hamburg, Staatsinstitut für Allgem. Botanik und Botanischer Garten) Afrikanische Pflanzen in Hamburg, Hamburger Botaniker in

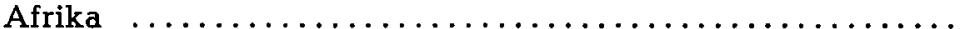

Oboussier, Henriette

(Universität Hamburg, Zoologisches Staatsinstitut und Zoologisches Museum) Der Anteil Hamburgs an der zoologischen Erforschung Afrikas

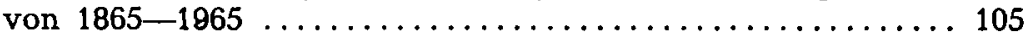


Asshauer, Egbert

(Bernhard-Nocht-Institut für Schiffs- und Tropenkrankheiten, Hamburg)

Die Beziehungen der Hamburger Tropenmedizin zu Afrika . . . 111

DitTMER, KunZ

(Hamburgisches Museum für Völkerkunde und Vorgeschichte)

Hamburger völkerkundliche Forschungen in Afrika

Lukas, Johannes und Mitarbeiter

(Universität Hamburg, Seminar für Afrikanische Sprachen und Kulturen)

Afrikanische Sprachen und Kulturen - der Hamburger Beitrag

zu ihrer Erforschung $\ldots \ldots \ldots \ldots \ldots \ldots \ldots \ldots \ldots \ldots \ldots \ldots$

Dammann, ERnST

(Universität Marburg, Afrikanische Abteilung des Orientalischen Seminars)

Hamburger Missionswissenschaft .............. 181

SCHRÖDER, Dieter

(Universität Hamburg, Forschungsstelle für Völkerrecht und ausländ.

Offentliches Recht)

Hamburger Beiträge zur Erforschung der Afrikanischen Rechts-

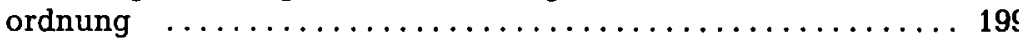

